\title{
Neue Low Version mit geringerer Bauhöhe
}

Die platzsparende Konstruktion von cara YantaLoc ${ }^{\circledR}$ sorgt bereits seit 1 Jahr für viel Freiheit bei der Prothesengestaltung. Jetzt hat Heraeus Kulzer mit cara YantaLoc LV durch reduzierte Abmessungen bei der Titanbasis und dem Halteelement den technischen Spielraum erneut entscheidend erweitert. cara YantaLoc ist eine verschraubte Titanbasis mit einem angulierten Zirkondioxid-Halteelement und integrierter LocatorFunktion. Es wurde konzipiert, um herausnehmbaren Zahnersatz einfach, sicher und flexibel auf Implantaten zu befestigen - auch bei geringen Platzverhältnissen im Patientenmund. Durch die niedrigere Bauhöhe ab 4,0 mm und einer Gingivahöhe von 1-2 mm, kann der Platzbedarf entscheidend reduziert werden. Zum Vergleich: Bisher war die niedrigste Version mit einer Bauhöhe ab 6,5 mm und einer Gingivahöhe von 1-3 mm erhältlich. Beide Varianten sind für alle gängigen Implantatsysteme lieferbar und können in 5 unterschiedlichen Angulationen von $0^{\circ}$ bis zu $20^{\circ}$ bestellt werden. Für zusätzliche Flexibilität ist es möglich, das Halteelement um $-60^{\circ}, 0^{\circ}$ oder $+60^{\circ}$ zur Implantatlage zu drehen. Die neue Low-Version ist ab sofort bei Heraeus Kulzer erhältlich. Anwender in Deutschland bestellen direkt über die kostenlose Bestellhotline unter 0800.437-2522, Kunden in Österreich über die Hotline des Customer Supports unter 0800.437-233. Weitere Informationen zu cara YantaLoc LV sowie die aktuelle Plattformübersicht finden Anwender unter www.heraeus-kulzer.de/cara-yantaloc.

Nach einer Pressemitteilung der Heraeus Kulzer GmbH, Hanau 